\title{
Gravity triggered neutrino condensates
}

\author{
Gabriela Barenboim \\ Departament de Física Teòrica and IFIC, Universitat de València-CSIC, E-46100, Burjassot, Spain.
}

(Dated: October 24, 2018)

\begin{abstract}
In this work we use the Schwinger-Dyson equations to study the possibility that an enhanced gravitational attraction triggers the formation of a right handed neutrino condensate, inducing dynamical symmetry breaking and generating a Majorana mass for the right handed neutrino at a scale appropriate for the see-saw mechanism. The composite field formed by the condensate phase could drive an early epoch of inflation.

We find that to the lowest order, the theory does not allow dynamical symmetry breaking. Nevertheless, thanks to the large number of matter fields in the model, the suppression by additional powers in $G$ of higher order terms can be compensated, boosting them up to their lowest order counterparts. This way chiral symmetry can be broken dynamically and the infrared mass generated turns out to be in the expected range for a successful see-saw scenario.
\end{abstract}

PACS numbers:

\section{INTRODUCTION}

The most commonly accepted theory about the origin and the evolution of our universe is doubtlessly the hot big bang model. Such a model is based on two crucial observations: the discovery of the expansion of the universe as depicted by the Hubble's law, and the existence of an astonishing isotropic and a perfectly thermal Cosmic Microwave Background (CMB) radiation. Since the energy density of radiation dilutes away faster with the expansion than that of matter, these two observations immediately point to the fact that the universe has evolved from a hot and dense early phase, when radiation, and not matter, was dominant. The recent evolution to a matter dominated epoch takes place when the radiation density falls sufficiently low that the photons cease to interact with matter. The CMB is then nothing but the remnant of a lost epoch, in which radiation was the dominant component of the energy budget, the relic radiation which is reaching us today from this epoch of decoupling. While reasonably isotropic, the CMB possesses small anisotropies (of about one part in $10^{5}$ ), and the observed pattern of the fluctuations in the CMB provides a straight snapshot of the universe at this epoch. The hot big bang model has been rather successful in predicting, say, the primordial abundances of the light elements in terms of a single parameter, namely the baryon-tophoton ratio, and the amount required to fit these observations beautifully agrees with the value that has been arrived at independently from the structure of the anisotropies in the CMB. Despite the success of the hot big bang model in explaining the results from different observations, the model has a serious drawback. Under this framework, the CMB photons arriving at us today from sufficiently widely separated directions in the sky could not have interacted at the time of decoupling. Nevertheless, one finds that the temperature of the CMB photons reaching us from any two diametrically opposite directions scarcely vary ${ }^{1}$.

Inflation [2], which refers to a period of accelerated expansion during the early stages of the radiation dominated epoch, provides a satisfactory resolution to abovementioned deficiencies of the hot big bang model. Actually, in addition to offering a graceful explanation for the extent of homogeneity and isotropy of the background universe, inflation also provides an attractive causal mechanism to generate the inhomogeneities superimposed upon it. The inflationary phase blows up the tiny quantum fluctuations present at the beginning of the epoch and transforms them into classical perturbations which leave their watermarks as anisotropies in the CMB. Subsequently these anisotropies act as seeds for the formation of the large scale structures that we observe at the present time as galaxies and clusters of galaxies. With the anisotropies in the CMB being measured to greater and greater precision, we have an unprecedented fertile ground to test the predictions of inflation. The simplest models of inflation driven by a single, slowly rolling scalar field, generically predict a nearly scale invariant spectrum of primordial perturbations, which seems to be in excellent agreement with the recent observations of the CMB.

Although the inflationary paradigm is widely accepted, most attempts to incorporate inflation into specific models of particle physics struggle with two weaknesses. (i) Parameters such as coupling constants must often be fine-tuned to extremely small values in order to avoid massive overproduction of density fluctuations. This can be traced back in a general sense to emanate from an exponential dependence of the vacuum energy density $V^{4}$ on the symmetry

\footnotetext{
${ }^{1}$ For an account of the impressive successes and the scarce weaknesses of the hot big bang model see, for example [1]
} 
breaking scale $V$. (ii) Symmetry breaking scales significantly below the Planck scale, $V<M_{P l} \simeq 10^{19} \mathrm{GeV}$ are at odds with observational constraints. The latter deficiency is particularly bothering, since physics at the Planck scale is at present poorly understood, and there is not a powerful basis to assume that standard notions of spontaneous symmetry breaking are valid at such high energies.

Models giving rise to inflation are typically formulated in terms of an elementary scalar field, the inflaton. In most models the inflaton field, $\phi$, is conceived as a scalar field (associated in the most ambitious models with a grand unified Higgs field) whose effective potential $V_{\text {eff }}(\phi)$ is chosen such that $\phi$ is initially trapped in a local minimum at $\phi=0$ (where it is specially flat) and has to roll down slowly to its true minimum at $\phi=\phi_{0}$. For a suitable choice of the model parameters the universe would spend a significant period of time in an exponentially expanding de Sitter phase, with its energy density dominated by the large value $V_{\text {eff }}(0)-V_{\text {eff }}\left(\phi_{0}\right)$. A crucial fine tuning is required in these models. Let alone the fact that these models reckon upon the existence of a fundamental scalar field.

As fundamental scalar fields are yet to be observed, in this work we explore the possibility of giving the inflaton a structure, as a dynamically generated condensate of right handed neutrinos triggered by gravitation.

Why neutrinos? Neutrinos have provided our first (and so far the only) glimpse beyond the Standard Model as neutrino oscillation experiments have shown unambiguous evidence that the three active neutrinos have mass and mix. Neutrino masses, as deduced from oscillation experiments point towards the existence of right handed neutrino states and a new energy scale. The new energy scale, is associated with the fact that the triviality of the quantum numbers of the new states allows them to have Majorana masses of order $M$, as well as to couple to the $S U(2)_{L}$ doublets and Higgs bosons. Although all values for $M$ are technically natural, large values of $M$ are preferred for several reasons, including the fact that one may relate $M$ to other well justified high energy scales, as $M_{P l}$ and $M_{G U T}$. Within the see-saw mechanism [3], a theoretically appealing scenario to generate the observed light masses in a natural way, the coupling to the Higgs doublet becomes a Dirac mass of order $\mu$ after electroweak symmetry breaking, while the Majorana masses are at some high scale, $M \sim M_{G U T}$. In this case, the resulting propagating neutrino degrees of freedom separate into two quasi-decoupled groups: mostly active states with masses $m \sim \mu^{2} / M$ and mostly sterile states with very large masses. In this case the right handed neutrinos will have super-heavy masses, as compared to their standard model counterparts. Being so heavy turns them into ideal candidates to provide structure (dynamical origin) to another super-heavy and weakly coupled particle, the inflaton.

Trying to form a right handed neutrino condensate, faces an obvious problem. Neutrinos are weakly inteacting particles. Even more, right handed neutrinos are solo players in the standard model, leaving gravity as their only possibility. Gravity, a force known to be miserably weak among elementary particles. So we want to explore the prospects of gravitational interactions between right handed neutrinos at ultra-high energies, i.e. in the very early universe, being strong enough to trigger the formation of a low-energy condesate, breaking lepton number and giving masses to right handed neutrinos.

As before, we can ask again, why neutrinos ? wouldn't this work (if it indeed works) for any other fermion as well ? If neutrinos do condensate, why doesn't it happen to say, electrons or quarks ? The answer to this puzzling behaviour rests on the fact that neutrinos are the only known fundamental neutral fermion. And this fact singles them out from the rest. For all charged fermions, when gauge interactions are attractive, a new annihilation channel opens up, closing the door to condensation. On the other hand, when annihilation is not possible, gauge interactions are repulsive.

\section{SETTING THE SCENE}

We will investigate whether chiral symmetry is broken dynamically due to the formation of a right handed neutrino condesate triggered by gravity. The formation of such a condensate, if it extists, sets off dynamical symmetry breaking of lepton number and produces a Majorana mass for the right-handed neutrino. The phenomenology of right handed neutrino condensates in the early universe was studied in [4] by introducing an effective four fermion self-coupling for the right handed neutrinos. It was found there that the condensate dynamics also produces "natural inflation" [5]. This way both, the inflationary de Sitter scale and the right handed neutrino mass, come out naturally of order of the infrared mass of the condensate. The quantum fluctuations of the condensate, which mimics a scalar field, give rise to primordial density fluctuations of the needed size and a spectral index in agreement with observations. The tensor perturbations however end up being exponentially suppressed, while the predictions for the running of the spectral index come out to be negligible small, experimentally indistinguishable from zero, making the model especially easy to test in the next generation of experiments. As compared to the usual approach to inflaton model building a dynamical framework is both economical and predictive. In this work we will try to relate the dynamics underlying the effective four fermion interaction to quantum gravity.

The Schwinger-Dyson equations provide a convenient way to study dynamical symmetry breaking and dynamical mass generation in quantum field theory. However, they constitute an infinite set of integral equations and some 
truncation scheme is necessary in order to explore its possible solutions.

Since quantum gravity is known to be non renormalizable, it will be necessary to introduce a momentum cut-off $\Lambda$ into the theory. Intuitively one assumes that $\Lambda$ is of order $M_{P l}$, Planck's mass. We will see that when the number of matter fields is large a lower, reduced Planck scale, will play an important role.

We can now explore dynamical symmetry breaking using the Schwinger-Dyson equation for the fermion propagator. Because of Lorentz and parity invariance the full propagator for the neutrino field $S_{F}$ can be written in the massless limit as

$$
S_{F}^{\prime}(p)=\frac{i}{\not p-\Sigma(p)}=\frac{i}{\alpha\left(p^{2}\right) \not p-\beta\left(p^{2}\right)}
$$

then the self energy part $\Sigma(p)$ satisfies the following integral equation

$$
\Sigma(p)=\int \frac{d^{4} k}{(2 \pi)^{4}} \Gamma_{\alpha \beta}(k-p, p) S_{F}(k) G^{\alpha \beta \mu \nu}(p-k) \Gamma_{\mu \nu}^{\prime}(p-k, k)
$$

where $\Gamma_{\mu \nu}^{(\prime)}(p, k)$ is the tree (full) fermion-fermion-graviton vertex function and $G^{\prime \alpha \beta \mu \nu}$ stands for the full graviton

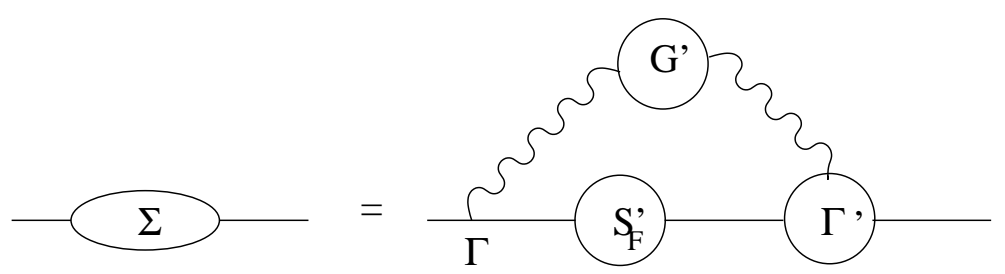

FIG. 1: The Schwinger-dyson equation for the fermion full propagator. Prime denotes full quantities

propagator. We will perform our calculation in the standard harmonic gauge and will consider an approximation where we replace $G^{\prime}$ and $\Gamma^{\prime}$ by their tree level values $G$ and $\Gamma$ respectively. Then, for the lowest order, we obtain the coupled integral equations

$$
\alpha\left(p^{2}\right)=1-i \kappa^{2} \int \frac{d^{4} k}{(2 \pi)^{4}} \frac{\alpha\left(k^{2}\right)}{\alpha\left(k^{2}\right) k^{2}-\beta^{2} k^{2}} \frac{1}{16(p-k)^{2} p^{2}}\left(4 k^{2} p^{2}+3\left(k^{2}+p^{2}\right) k p+2(k p)^{2}\right)
$$

where $\kappa^{2}=32 \pi G$ and

$$
\beta\left(p^{2}\right)=i \kappa^{2} \int \frac{d^{4} k}{(2 \pi)^{4}} \frac{\beta\left(k^{2}\right)}{\alpha\left(k^{2}\right) k^{2}-\beta^{2} k^{2}} L(p, k)
$$

Within the approximations we are using, and at the lowest order $L(p, k)=0$ and therefore $\beta=0$ which implies no symmetry breaking at all. We are forced then to go beyond the lowest order, the bare graviton, if we are to find a non-trivial solution for $\beta$. By going to the next leading term, we will obtain terms which are higher order in $G$ and therefore we will include them only for $\beta$ and not for $\alpha$ where the bare propagator contribution is the dominant one.

The standard model possesses a large number of matter degrees of freedom: 12 gauge bosons, 48 chiral fermions (including right handed neutrinos) and 4 Higgs scalars. In extensions of the standard model the matter content is even larger. This large number of matter degrees of freedom can help overcome the additional $G$ suppression factor arising with the next leading contribution. However, not all the one loop diagrams enjoy this large $N$ increase. We will consider only the vacuum polarization diagrams (corrections to the propagator), and not vertex corrctions, as they are the only ones who benefit from the large matter content of the theory close to the Planck scale and will exhibit a large $N$ enhancement.

The fact that $L(p, k)=0$ and therefore the chiral symmetry is unbroken at tree level is indeed remarkable. This may be related to gravity's structure itself, although one could question whether this is an artifact of the standard gauge we have chosen.

The matter contribution to the graviton self-energy is obtained from the subdiagrams in Fig. 2 and includes the one loop contributions for gauge bosons, Dirac fermions, minimal scalars, conformal scalars and gravitons.

The contribution of the photon/gauge boson loop to the polarization tensor reads

$$
\begin{aligned}
\tilde{\Pi}_{\alpha \beta, \gamma \delta}= & \frac{G}{\pi}\left[\frac{1}{30}\left(q_{\alpha} q_{\beta}-q^{2} \eta_{\alpha \beta}\right)\left(q_{\gamma} q_{\delta}-q^{2} \eta_{\gamma \delta}\right)-\frac{1}{20}\left(q_{\alpha} q_{\gamma}-q^{2} \eta_{\alpha \gamma}\right)\left(q_{\beta} q_{\delta}-q^{2} \eta_{\beta \delta}\right)\right. \\
& \left.-\frac{1}{20}\left(q_{\alpha} q_{\delta}-q^{2} \eta_{\alpha \delta}\right)\left(q_{\beta} q_{\gamma}-q^{2} \eta_{\beta \gamma}\right)\right] \log \left(\mu^{2} /-q^{2}\right),
\end{aligned}
$$




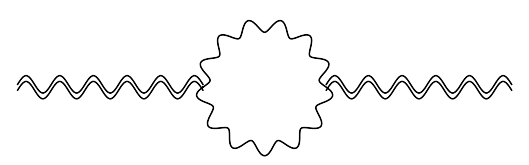

(a)

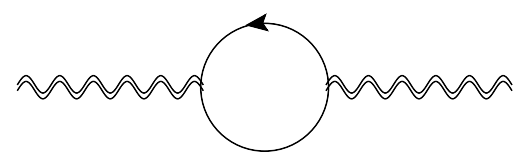

(b)

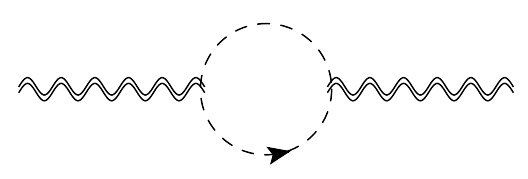

(c)

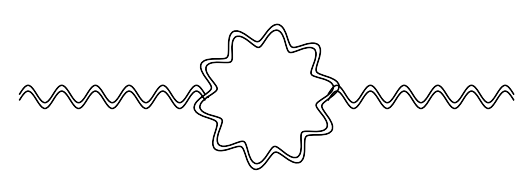

(d)

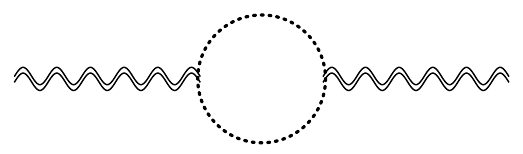

(e)

FIG. 2: The set of vacuum polarization diagrams which contribute to the graviton self-energy, which comprises gauge bosons (a), Dirac fermions (b), minimal and conformal scalars (c) and gravitons (d). Notice that there exists a ghost diagram (e) along with the graviton one.

where $q=p-k$ and we have introduced a renormalization mass $\mu$ which is in principle arbitrary. The gauge boson loop yields as a contribution to the kernel $L(p, k)$ of the $\beta$ term for the self energy of the graviton

$$
L_{p h}(p, k)=\tau^{\mu \nu}\left[\frac{\mathcal{P}_{\mu \nu \alpha \beta}}{q^{2}}\right] \tilde{\Pi}^{\alpha \beta, \gamma \delta}(q)\left[\frac{\mathcal{P}_{\gamma \delta \rho \sigma}}{q^{2}}\right] \tau^{\rho \sigma}=-\frac{G^{2}}{3}\left[(p+k)^{2}-\frac{\left(p^{2}-k^{2}\right)^{2}}{(p-k)^{2}}\right] \log \left(\frac{\mu^{2}}{-(p-k)^{2}}\right)
$$

where $\mathcal{P}_{\mu \nu \alpha \beta}$ is the polarization tensor, defined in the appendix.

We have to include also the Dirac fermion loop (Majorana fermion contribution halves)

$$
\begin{aligned}
\hat{\Pi}_{\alpha \beta, \gamma \delta}= & \frac{G}{\pi}\left[\frac{1}{60}\left(q_{\alpha} q_{\beta}-q^{2} \eta_{\alpha \beta}\right)\left(q_{\gamma} q_{\delta}-q^{2} \eta_{\gamma \delta}\right)-\frac{1}{40}\left(q_{\alpha} q_{\gamma}-q^{2} \eta_{\alpha \gamma}\right)\left(q_{\beta} q_{\delta}-q^{2} \eta_{\beta \delta}\right)\right. \\
& \left.-\frac{1}{40}\left(q_{\alpha} q_{\delta}-q^{2} \eta_{\alpha \delta}\right)\left(q_{\beta} q_{\gamma}-q^{2} \eta_{\beta \gamma}\right)\right] \log \left(\mu^{2} /-q^{2}\right),
\end{aligned}
$$

whose addition to the $\beta$ term input to the self energy gives

$$
L_{d f}(p, k)=\tau^{\mu \nu}\left[\frac{\mathcal{P}_{\mu \nu \alpha \beta}}{q^{2}}\right] \hat{\Pi}^{\alpha \beta, \gamma \delta}(q)\left[\frac{\mathcal{P}_{\gamma \delta \rho \sigma}}{q^{2}}\right] \tau^{\rho \sigma}=-\frac{G^{2}}{6}\left[(p+k)^{2}-\frac{\left(p^{2}-k^{2}\right)^{2}}{(p-k)^{2}}\right] \log \left(\frac{\mu^{2}}{-(p-k)^{2}}\right)
$$

And also minimal scalar fields

$$
\begin{aligned}
\bar{\Pi}_{\alpha \beta, \gamma \delta}= & \frac{G}{\pi}\left[\frac{1}{40}\left(q_{\alpha} q_{\beta}-q^{2} \eta_{\alpha \beta}\right)\left(q_{\gamma} q_{\delta}-q^{2} \eta_{\gamma \delta}\right)-\frac{1}{240}\left(q_{\alpha} q_{\gamma}-q^{2} \eta_{\alpha \gamma}\right)\left(q_{\beta} q_{\delta}-q^{2} \eta_{\beta \delta}\right)\right. \\
& \left.-\frac{1}{240}\left(q_{\alpha} q_{\delta}-q^{2} \eta_{\alpha \delta}\right)\left(q_{\beta} q_{\gamma}-q^{2} \eta_{\beta \gamma}\right)\right] \log \left(\mu^{2} /-q^{2}\right),
\end{aligned}
$$

that contribute to the self energy and thus to the kernel of the $\beta$ term by

$$
L_{m s}(p, k)=\tau^{\mu \nu}\left[\frac{\mathcal{P}_{\mu \nu \alpha \beta}}{q^{2}}\right] \breve{\Pi}^{\alpha \beta, \gamma \delta}(q)\left[\frac{\mathcal{P}_{\gamma \delta \rho \sigma}}{q^{2}}\right] \tau^{\rho \sigma}=-\frac{3 G^{2}}{8}\left[(p+k)^{2}-\frac{2}{3} \frac{\left(p^{2}-k^{2}\right)^{2}}{(p-k)^{2}}\right] \log \left(\frac{\mu^{2}}{-(p-k)^{2}}\right)
$$

Last but not least we have to include also the contribution of conformal scalars

$$
\begin{aligned}
\breve{\Pi}_{\alpha \beta, \gamma \delta}= & \frac{G}{\pi}\left[\frac{1}{360}\left(q_{\alpha} q_{\beta}-q^{2} \eta_{\alpha \beta}\right)\left(q_{\gamma} q_{\delta}-q^{2} \eta_{\gamma \delta}\right)-\frac{1}{240}\left(q_{\alpha} q_{\gamma}-q^{2} \eta_{\alpha \gamma}\right)\left(q_{\beta} q_{\delta}-q^{2} \eta_{\beta \delta}\right)\right. \\
& \left.-\frac{1}{240}\left(q_{\alpha} q_{\delta}-q^{2} \eta_{\alpha \delta}\right)\left(q_{\beta} q_{\gamma}-q^{2} \eta_{\beta \gamma}\right)\right] \log \left(\mu^{2} /-q^{2}\right)
\end{aligned}
$$


whose addition to the kernel of the $\beta$ function coming from their contribution to the self energy yields

$$
L_{c s}(p, k)=\tau^{\mu \nu}\left[\frac{\mathcal{P}_{\mu \nu \alpha \beta}}{q^{2}}\right] \bar{\Pi}^{\alpha \beta, \gamma \delta}(q)\left[\frac{\mathcal{P}_{\gamma \delta \rho \sigma}}{q^{2}}\right] \tau^{\rho \sigma}=-\frac{G^{2}}{36}\left[(p+k)^{2}-\frac{\left(p^{2}-k^{2}\right)^{2}}{(p-k)^{2}}\right] \log \left(\frac{\mu^{2}}{-(p-k)^{2}}\right)
$$

Despite not being a matter field, the graviton loop contribution to its self energy could prove to be important, although subdominant in a formal large $N$ expansion. Its contribution to the effective Lagrangian has been worked out by 't Hooft and Veltman [6], and from it one can estimate its contribution to the vacuum polarization tensor

$$
\begin{aligned}
\Pi_{\alpha \beta, \gamma \delta}= & \frac{G}{\pi}\left[\frac{23}{60}\left(q_{\alpha} q_{\beta}-q^{2} \eta_{\alpha \beta}\right)\left(q_{\gamma} q_{\delta}-q^{2} \eta_{\gamma \delta}\right)-\frac{7}{40}\left(q_{\alpha} q_{\gamma}-q^{2} \eta_{\alpha \gamma}\right)\left(q_{\beta} q_{\delta}-q^{2} \eta_{\beta \delta}\right)\right. \\
& \left.-\frac{7}{40}\left(q_{\alpha} q_{\delta}-q^{2} \eta_{\alpha \delta}\right)\left(q_{\beta} q_{\gamma}-q^{2} \eta_{\beta \gamma}\right)\right] \log \left(\mu^{2} /-q^{2}\right)
\end{aligned}
$$

Inserting this expression into the self energy, from the graviton loop one obtains the following contribution to the kernel of the $\beta$-term

$$
L_{g r}(p, k)=\tau^{\mu \nu}\left[\frac{\mathcal{P}_{\mu \nu \alpha \beta}}{q^{2}}\right] \Pi^{\alpha \beta, \gamma \delta}(q)\left[\frac{\mathcal{P}_{\gamma \delta \rho \sigma}}{q^{2}}\right] \tau^{\rho \sigma}=-8 G^{2}\left[\frac{89}{96}(p+k)^{2}-\frac{31}{48} \frac{\left(p^{2}-k^{2}\right)^{2}}{(p-k)^{2}}\right] \log \left(\frac{\mu^{2}}{-(p-k)^{2}}\right)
$$

Including all these contributions we obtain

$$
\begin{aligned}
\beta\left(p^{2}\right) & =i 2 \pi G^{2} \int \frac{d^{4} k}{(2 \pi)^{4}} \frac{\beta\left(k^{2}\right)}{\alpha\left(k^{2}\right) k^{2}-\beta^{2} k^{2}}\left[A(p+k)^{2}-B \frac{\left(p^{2}-k^{2}\right)^{2}}{(p-k)^{2}}\right] \log \left(\frac{\mu^{2}}{-(p-k)^{2}}\right) \\
& =i 2 \pi G^{2} \int \frac{d^{4} k}{(2 \pi)^{4}} \frac{\beta\left(k^{2}\right)}{\alpha\left(k^{2}\right) k^{2}-\beta^{2} k^{2}}\left\{A\left[(p+k)^{2}-\frac{\left(p^{2}-k^{2}\right)^{2}}{(p-k)^{2}}\right]+C \frac{\left(p^{2}-k^{2}\right)^{2}}{(p-k)^{2}}\right\} \log \left(\frac{\mu^{2}}{-(p-k)^{2}}\right)
\end{aligned}
$$

with

$$
\begin{aligned}
A & =-\frac{267+12 N_{g b}+6 N_{d f}+27 / 2 N_{m s}+N_{c s}}{288} \\
B & =\frac{186+12 N_{g b}+6 N_{d f}+9 N_{m s}+N_{c s}}{288} \\
C & =\frac{81+9 / 2 N_{m s}}{288}
\end{aligned}
$$

where $N_{g b}, N_{d f}, N_{m s}$ and $N_{c s}$ correspond to the number of gauge bosons, Dirac fermions, minimal scalar and conformal scalar degrees of freeedom in the model, respectively.

In eq.(16) the conformal contributions are contained in the $A$ term while conformal breaking terms contribute to the $C$ term. While both terms can help trigger dynamical symmetry breaking, the $A$ term for small external moment, $p \ll k$, becomes

$$
\left[(p+k)^{2}-\frac{\left(p^{2}-k^{2}\right)^{2}}{(p-k)^{2}}\right] \stackrel{p \ll k}{\longrightarrow}-(k \cdot p)^{2}+4 k^{2} p^{2} \longrightarrow 0
$$

so that it cannot give rise to an infrared mass. Thus, it is only the term proportional to $C$ that can provide a dynamical infrared mass to the right handed neutrino, effectively breaking both symmetries, lepton number and chiral symmetry, dynamically. The infrared mass generated will be supressed with respect to the cut-off scale by the factor $C / A$ which is $\mathcal{O}\left(1 / N_{\text {matter }}\right)$.

Now imposing $\alpha$ positive and $\beta$ real for space-like momentum, which is imposing that the fermion is stable without a tachyonic singularity, we can safely perform the angular integration after the Wick rotation. We get

$$
\alpha(x)=1-\frac{G \Lambda^{2}}{32 \pi} \int_{0}^{1} d y K(x, y) \frac{y \alpha(y)}{y \alpha^{2}(y)+\beta^{2}(y)}
$$

and

$$
\beta(x)=\frac{G^{2} \Lambda^{4}}{16 \pi} \int_{0}^{1} d y L(x, y) \frac{y \beta(y)}{y \alpha^{2}(y)+\beta^{2}(y)}
$$


where $x=p^{2} / \Lambda^{2}$ and $y=k^{2} / \Lambda^{2}$ and

$$
\begin{aligned}
K(x, y)= & \frac{1}{x}\left[\frac{16\left(x^{2}+y^{2}\right)+64 x y}{x+y+|x-y|}-8(x+y)\right] \\
L(x, y)= & \frac{A}{48 x y}\left\{-2|x-y|\left(5 x^{2}+2 x y+5 y^{2}\right)+(x+y)\left(-36 x y+10(x+y)^{2}\right)+24 x y(x+y) \log \left[\frac{x+y+|x-y|}{2}\right]\right\} \\
& +\frac{B}{8 x y}\left\{2|x-y|\left[1-2 \log \left[\frac{2(x-y)^{2}}{x+y+|x-y|}\right]\right]+(x+y)\left[-2+\log \left[\frac{-2 x y+(x+y)(x+y+|x-y|)}{2}\right]\right]\right\}
\end{aligned}
$$

We have introduced an UV momentum cut-off $\Lambda$, arising from the non-renormalizabilty of the theory which does not necessarily possess the same value as the scale $\mu$. However one would expect them to be of the same order of magnitude. In the remaining of this work we will consider them to be equal, $\Lambda=\mu=0.8 M_{P l}$.

As in [7], we implement the following iterative method to find the numerical solutions of the Schwinger-Dyson equations. We first choose two trial functions to start our iterative calculation

$$
\alpha^{(0)}(x)=c_{1} \quad, \beta^{(0)}(x)=c_{2}
$$

with $c_{1}$ and $c_{2}$ constants. We then define $\alpha^{(i)}$ and $\beta^{(i)}$ by

$$
\alpha^{(i+1)}(x)=1-\frac{G \Lambda^{2}}{32 \pi} \int_{0}^{1} d y K(x, y) \frac{y \alpha^{(i)}(y)}{y \alpha^{(i) 2}(y)+\beta^{(i) 2}(y)}
$$

and

$$
\beta^{(i+1)}(x)=\frac{G^{2} \Lambda^{4}}{16 \pi} \int_{0}^{1} d y L(x, y) \frac{y \beta^{(i)}(y)}{y \alpha^{(i) 2}(y)+\beta^{(i) 2}(y)}
$$

expecting that the series $\alpha^{(i)}(x)$ and $\beta^{(i)}(x)$ converge into the solutionts of the Schwinger-Dyson equations $\alpha(x)$ and $\beta(x)$, respectively. In practice, we assume that the series $\alpha^{(i)}(x)$ and $\beta^{(i)}(x)$ converge if the normalized difference $\left|\alpha^{(i+1)}-\alpha^{(i)}\right|+\left|\beta^{(i+1)}-\beta^{(i)}\right|$ become less than $10^{-3}$.

For a true dynamical symmetry breaking one has to check that the minimum we have found is a real minimum. For that purpose, we use the effective potential defined in [11]. In the Hartree-Fock approximation the effective potential $V[S]$ as the functional of the fermion propagator $S$ is obtained to be

$$
\begin{aligned}
V[S]= & -i \int \frac{d^{4} p}{(2 \pi)^{4}} \operatorname{Tr}\left[\log S_{F}^{-1}(p) S(p)-S_{F}^{-1}(p) S(p)+1\right] \\
& -\frac{i}{2} \int \frac{d^{4} p d^{4} k}{(2 \pi)^{8}} \operatorname{Tr}\left[\Gamma_{\alpha \beta}(k, p-k) S(p) \Gamma_{\mu \nu}(p, k-p) S(k) G^{\alpha \beta \mu \nu}(k-p)\right]
\end{aligned}
$$

Substituting the solution of the Swinger-Dyson equation $\delta V\left[S_{F}^{\prime}\right] / \delta S_{F}^{\prime}=0$ into the above expression we get

$$
V\left[S_{F}\right]=\frac{-i}{2} \int \frac{d^{4} p}{(2 \pi)^{4}} \operatorname{Tr}\left[2 \log S_{F}^{-1}(p) S_{F}^{\prime}(p)-S_{F}^{-1}(p) S_{F}^{\prime}(p)+1\right]
$$

Using now that $S_{F}(p)=i / \not p$ and eq(1) and performing the angular integration after Wick's rotation we have

$$
V[\alpha, \beta]=\frac{\Lambda^{4}}{8 \pi} \int_{0}^{1} d x\left\{\log \frac{x}{x \alpha^{2}(x)+\beta^{2}(x)}-\frac{x \alpha(x)}{x \alpha^{2}(x)+\beta^{2}(x)}+1\right\}
$$

where $\alpha$ and $\beta$ are the solutions of eqs (21)-(22). As the solution corresponding to the true vacuum should minimize the potential, if there is a symmetry breaking solution $\left(\alpha=\alpha_{\beta}, \beta \neq 0\right)$ besides the symmetry conserving solution $\left(\alpha=\alpha_{o}, \beta=0\right)$ a difference of potentials

$$
\delta V=V\left[\alpha=\alpha_{\beta}, \beta \neq 0\right]-V\left[\alpha=\alpha_{o}, \beta=0\right]
$$

selects the solution of the two corresponding to a true vacuum.

In the standard model $A=203 / 96 \approx 2.1$ which is not large enough to produce a symmetry breaking minimum. A much larger matter content would be needed such that $A>8$ for that purpose. Cut-off scales even closer to $M_{P l}$ 
would allow symmetry breaking for smaller $A$. In minimal supersymmetry, with 12 gauge bosons, 80 chiral fermions and 56 real (minimal) scalars, $A$ turns out to be $A \approx 5$. The particle content again is not enough to produce symmetry breaking. However, extended models or models with extra dmensions, can reach values of $A$ in the desired range. For example in $\mathrm{N}=8$ supergravity [12] $A>8$. In Fig 3 we show the symmetry breaking solution of the Schwinger-Dyson equation for $A=10$.

Fig 4 shows our solution for $\beta(x)$ versus $x=p^{2} / \Lambda^{2}$ for values of momenta larger than the cut-off scale. It is evident there that the cut-off signals when the interaction stops being attactive. In this sense, its existence seems justified.

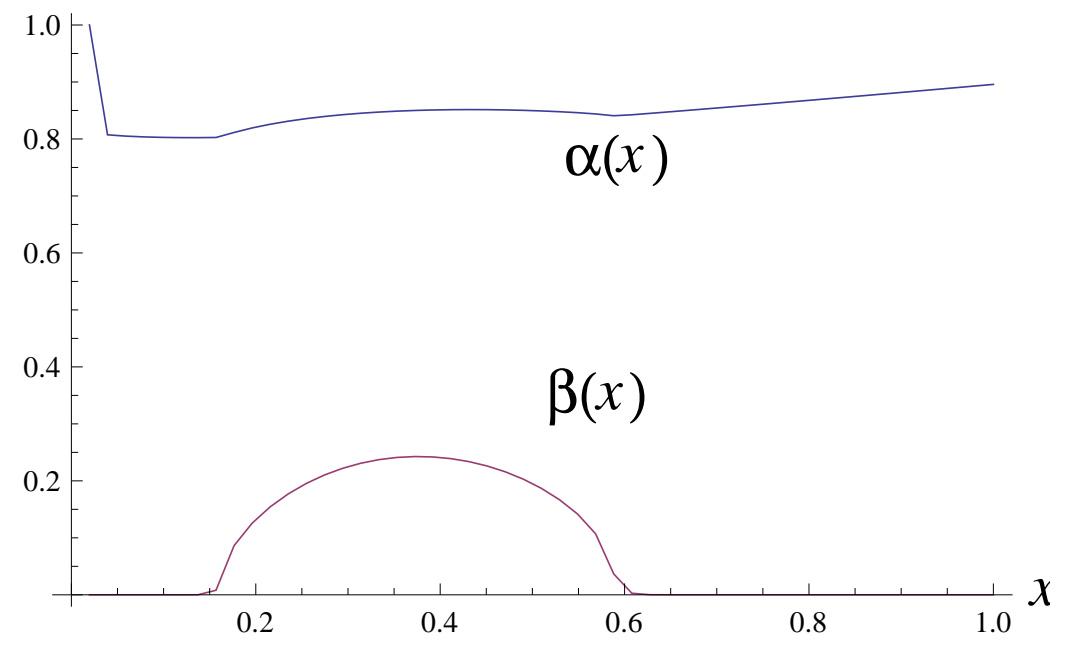

FIG. 3: The symmetry breaking solutions of the Schwinger-Dyson equation, $\alpha$ and $\beta$ for $A=10$ as a function of $x=p^{2} / \Lambda^{2}$

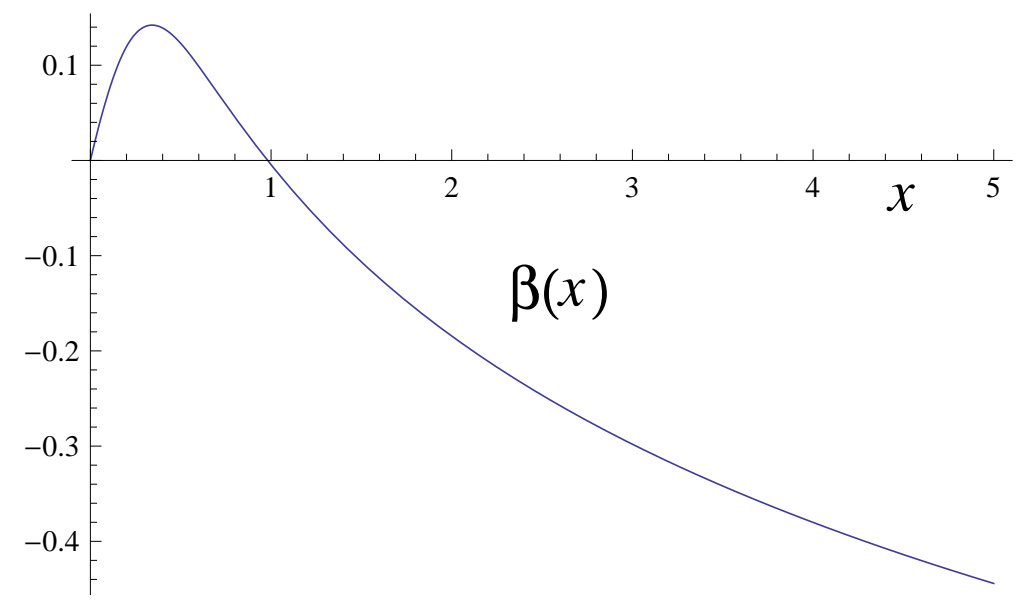

FIG. 4: The solution of the Schwinger-Dyson equation $\beta(x)$ becomes repulsive for momenta larger than the cut-off scale $\Lambda$

Once the symmetry is broken a mass for the right handed neutrino will be produced. Such a mass, arises from the conformal breaking part of the graviton or the minimal scalar loop contributions (the $C$ term), and is roughly given by

$$
m_{R H} \approx \frac{C}{A} \frac{\Lambda}{4 \pi} \sim \mathcal{O}(1 / N) .
$$

where $N$ reflects matter field contribution to the conformal term. For the successful symmetry breaking scenario shown before, with $A=10$, it produces a mass $m_{R H} \approx 10^{-3} \Lambda$, exactly in the right ball-park to a succesful see-saw. 


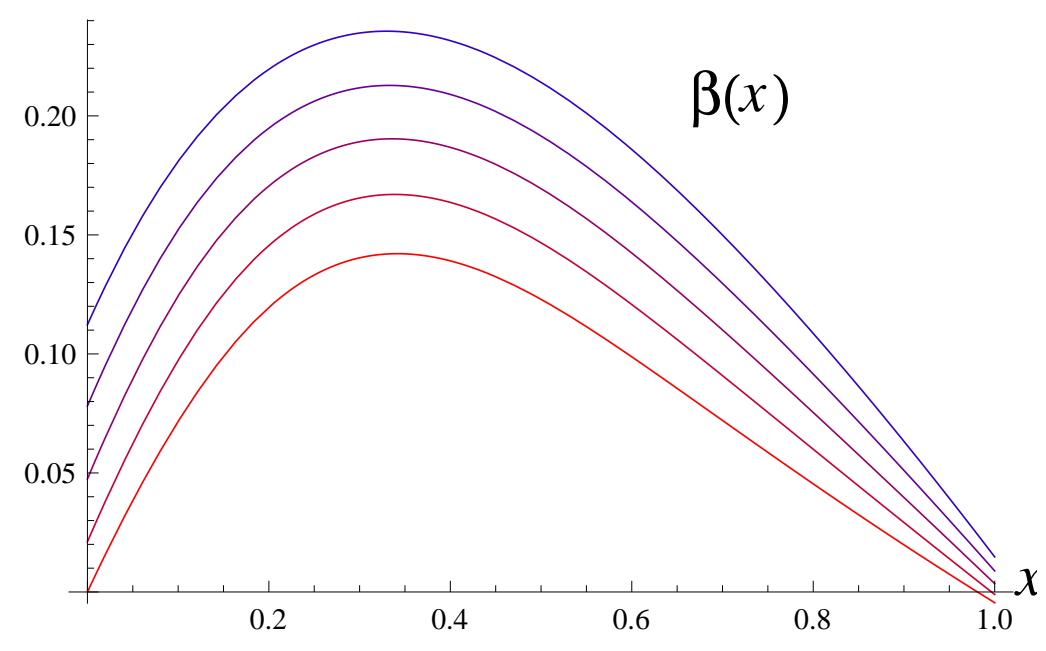

FIG. 5: $\beta(x)$ for $C / A=0.2$ (red), 0.4, $0.6,0.8,1$ (blue) as a function of $x=p^{2} / \Lambda^{2}$ The larger the ratio, the heavier the infrared mass

\section{RESUMMATION}

As we have seen in the previous section, the large matter content of the standard model (and the even larger one of extended theories) could help overcome the $G$ suppression of the next leading order contribution to the kernel of the $\beta$ function, opening the door to a dynamical symmetry breaking. If this is the case, then some additional, higher order contributions, suppressed, in principle by extra powers of $G$ would also benefit from the $N$ enhancement and must be included in the calculation.

Following Tomboulis [8] we can include those contributions. As $N$ (the number of matter fields) becomes large (even if not as large and we would have needed in the previous section), a natural expansion would be then an expansion in powers of $1 / N$, keeping $G N$ fixed. Hence, we will explore the $1 / N$ expansion where some systematic resumation of the perturbative series can be done.

The general validity of this $1 / N$ expansion is based on the fact that gravity couples universally to all matter. Besides, the expansion possesses important advantages which make it very convenient to use. Unlike the coupling $G, N$ is a scale independent parameter. Therefore, the solution to the theory to a given order in $1 / N$ as given by the expansion holds in principle for all momenta scales, and can be used to probe the strong coupling regime. Furthermore, being $N$ a gauge invariant parameter, the expansion is manifestly gauge invariant.

To proceed with the expansion, notice that to leading order in $1 / N$ we should include only those graphs which contain closed matter loops including fermions, gauge bosons or conformaly coupled scalars. The large number of matter fields defines a large $N$ enhancement that compensates for the Planck suppression of the additional coupling factor $G$ producing an effective "reduced" Planck mass, $M_{P l}^{2}$ reduced $=M_{P l}^{2} / N$. In fact the enhancement occurs only for the spin 2 part of the propagator, the $A$ term and not the trace part as reflected in the $C$ term. It is not necessary to include the vertex corrections as they enter only at the next level in the $1 / N$ expansion.

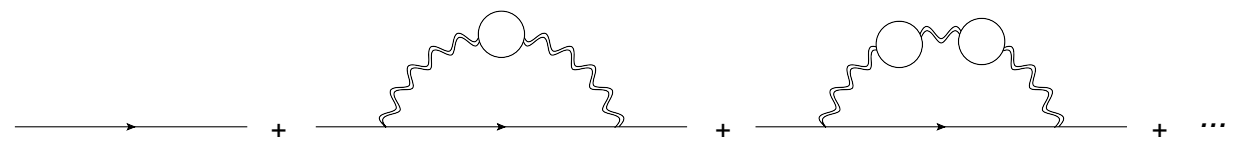

FIG. 6: Only those graphs which contain a closed loop of matter fields benefit from the large $N$ enhancement and are to be resummed

Therefore, the matter, gauge boson and conformally coupled scalar loop corrections to the bare graviton propagator must now be included as part of the leading order graviton propagator. Using the one loop contribution of such 
particles to the graviton self energy calculated before we obtain for the complete leading order graviton propagator

$$
D_{\mu \nu \alpha \beta}=\frac{\mathcal{P}_{\mu \nu \alpha \beta}^{(2)}}{q^{2}\left(1-\frac{2}{\pi} N G q^{2} \log \left(\frac{-q^{2}}{\mu^{2}}\right)\right)}
$$

where $\mathcal{P}^{(2)}$ is the spin 2 part of the propagator and $N$ is a measure of the number of matter fields in the theory, $N \propto N_{g b}+N_{d f} / 2+N_{(c s+m s)} / 12$. In Fig 7 the potential enhancement of the spacelike propagator due to large $N$ is depicted. Such enhancement is due to the fact that the leading $1 / N$ propagator posseses no spacelike spin 2 poles for real momenta. Instead, it posseses pairs of complex conjugate poles in the complex plane. As shown by

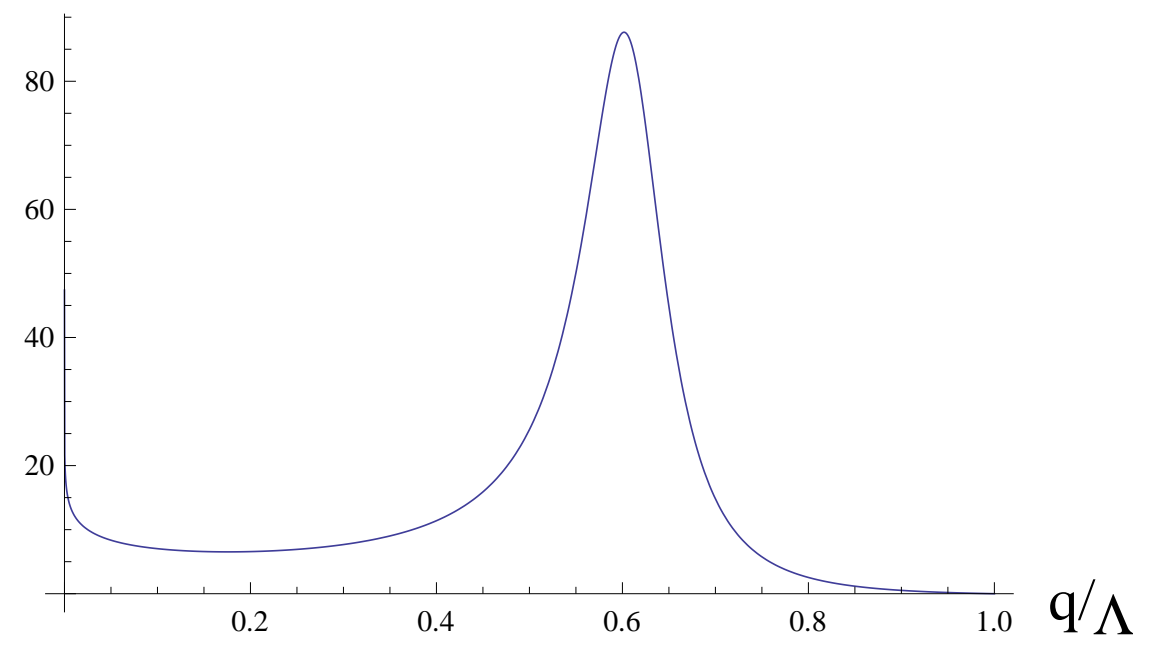

FIG. 7: Large $N$ enhancement of the space-like propagator in arbitrary units as a function of the momentum trasfer (in units of the cut-off $\Lambda$ )

Tomboulis [8, 9] , the gravitational quantum corrections modify the propagator in such a way that the new propagator has no unphysical poles on the real $q^{2}$ axis. The would-be unphysical poles are shifted off the real axis by the matter interactions and split into a pair of complex conjugate poles, i.e. quantum corrections shift the tachyonic ghost poles to a pair of complex poles on the physical sheet. According to Lee and Wick [10] the theory can be defined so that the complex poles do not contribute to the absorptive part of the amplitudes and physical unitarity can be maintained. However, the standard analyticity properties of the $S$ matrix are modified by the extra complex poles which results in a breakdown of locality. This should come as no surprise as we expect gravity to be non-local once quantum effects become important.

With this interpretation we can proceed with the resummation. We begin by re-writting the different contributions to the polarization tensor as a traceless spin 2 and a spin 0 part

$$
\begin{aligned}
\Pi_{\alpha \beta, \gamma \delta}= & \frac{\Pi_{2}}{q^{4}}\left[\left(q_{\alpha} q_{\gamma}-q^{2} \eta_{\alpha \gamma}\right)\left(q_{\beta} q_{\delta}-q^{2} \eta_{\beta \delta}\right)+\left(q_{\alpha} q_{\delta}-q^{2} \eta_{\alpha \delta}\right)\left(q_{\beta} q_{\gamma}-q^{2} \eta_{\beta \gamma}\right)-\right. \\
& \left.\frac{2}{3}\left(q_{\alpha} q_{\beta}-q^{2} \eta_{\alpha \beta}\right)\left(q_{\gamma} q_{\delta}-q^{2} \eta_{\gamma \delta}\right)\right]_{2}+\frac{\Pi_{o}}{q^{4}}\left[\left(q_{\alpha} q_{\beta}-q^{2} \eta_{\alpha \beta}\right)\left(q_{\gamma} q_{\delta}-q^{2} \eta_{\gamma \delta}\right)\right]_{0}
\end{aligned}
$$

define

$$
\hat{\eta}_{\mu \nu} \equiv q_{\mu} q_{\nu}-q^{2} \eta_{\mu \nu}
$$

and sum all the bubbles

$$
\begin{aligned}
D_{\mu \nu \alpha \beta}= & \frac{P}{q^{2}}+\frac{P}{q^{2}} \Pi \frac{P}{q^{2}}+\frac{P}{q^{2}} \Pi \frac{P}{q^{2}} \Pi \frac{P}{q^{2}}+\frac{P}{q^{2}} \Pi \frac{P}{q^{2}} \Pi \frac{P}{q^{2}} \Pi \frac{P}{q^{2}}+\ldots \\
& \frac{P}{q^{2}}+\frac{\Pi_{2}}{\left(q^{2}\right)^{2}} \frac{1}{1-2 \frac{\Pi_{2}}{q^{2}}}\left[\hat{\eta}_{\mu \alpha} \hat{\eta}_{\nu \beta}+\hat{\eta}_{\nu \alpha} \hat{\eta}_{\mu \beta}-\frac{2}{3} \hat{\eta}_{\alpha \beta} \hat{\eta}_{\mu \nu}\right]+\frac{\Pi_{0}}{\left(q^{2}\right)^{2}} \frac{1}{1+\frac{3}{2} \frac{\Pi_{0}}{q^{2}}}\left[\left(\hat{\eta}_{\mu \nu}-\frac{3}{2} \eta_{\mu \nu}\right)\left(\hat{\eta}_{\alpha \beta}-\frac{3}{2} \eta_{\alpha \beta}\right)\right]
\end{aligned}
$$


where $P$ is the graviton polarization sum $\mathcal{P}^{\mu \nu \alpha \beta}$ in the harmonic gauge and $\Pi$ represents the polarization tensor (Lorentz indices have been omitted). Then the resummed kernel becomes

$$
L_{r s}(p, k)=\tau_{\mu \nu} D^{\mu \nu \alpha \beta} \tau_{\alpha \beta}=\frac{8 \pi G}{\left(q^{2}\right)^{2}}\left\{\frac{\Pi_{2}}{1-2 \frac{\Pi_{2}}{q^{2}}} \frac{5}{6}\left[(p+k)^{2}-\frac{\left(p^{2}-k^{2}\right)^{2}}{(p-k)^{2}}\right]+\frac{\Pi_{0}}{1+\frac{3}{2} \frac{\Pi_{0}}{q^{2}}}\left[\frac{25}{16}(p+k)^{2}-\frac{\left(p^{2}-k^{2}\right)^{2}}{(p-k)^{2}}\right]\right\}
$$

with

$$
\Pi_{2}\left(q^{2}\right)=-\frac{G}{\pi}\left(q^{2}\right)^{2} \log \left(\frac{\mu^{2}}{-q^{2}}\right)\left[\frac{7}{40}+\frac{1}{20} N_{g b}+\frac{1}{40} N_{d f}+\frac{1}{240} N_{m s}+\frac{1}{240} N_{c s}\right]
$$

and

$$
\Pi_{0}\left(q^{2}\right)=-\frac{G}{\pi}\left(q^{2}\right)^{2} \log \left(\frac{\mu^{2}}{-q^{2}}\right)\left[\frac{1}{2}+\frac{1}{36} N_{m s}\right]
$$

with this the spin 2 denominator takes the form

$$
1-2 \frac{\Pi_{2}\left(q^{2}\right)}{q^{2}}=1+\frac{2 G}{\pi} q^{2} \log \left(\frac{\mu^{2}}{-q^{2}}\right)\left[\frac{7}{40}+\frac{1}{20} N_{g b}+\frac{1}{40} N_{d f}+\frac{1}{240} N_{m s}+\frac{1}{240} N_{c s}\right]
$$

while that of the spin 0 denominator gives

$$
1+\frac{3}{2} \frac{\Pi_{0}}{q^{2}}=1-\frac{3}{2} \frac{2 G}{\pi} q^{2} \log \left(\frac{\mu^{2}}{-q^{2}}\right)\left[\frac{1}{2}+\frac{1}{36} N_{m s}\right]
$$

notice that although both denominators go like $\frac{q^{2}}{\pi} \log \left(\frac{\mu^{2}}{q^{2}}\right)$, only the spin 2 part, the $A$ term, has the large $N$ enhancement. The large enhancement shown in Fig 7 occurs when the complex poles are close to the real axis as would be expected near criticality in the Lee-Wick theory. Thus enhancement is achieved by tuning the reduced Planck scale, i.e. by tuning the matter content of the theory and keeping the renormalization scale $\mu$ always below but not far from its maximum possible value $\mu_{\max }=\sqrt{e} M_{P l \text { reduced }}$.

In Fig 8 we show how the poles travel the complex plane as a function of the renormalizaton scale $\mu$. The spin 0 part, the $C$ term, even if close to the phase transition point does not get enhanced in the large $N$ scenario. The vast increase displayed in Fig [ is attained when the maximum of the $q^{2} \log \left(\frac{\mu^{2}}{q^{2}}\right)$ term in the denominator of the $A$ term is tuned to be close to 1 .

One can then expand the $\frac{q^{2}}{\pi} \log \left(\frac{\mu^{2}}{q^{2}}\right)$ term around its maximum, $Q^{2}=\mu^{2} / e$, as in the narrow width approximation, i.e. we can approximate the propagator shown before by a gaussian, so that we can estimate the size (the strength) of the spin 2 contribution, while neglecting that of the spin 0 part. For the spin-2 denominator we get

$$
\begin{aligned}
1-2 \frac{\Pi_{2}\left(q^{2}\right)}{q^{2}}= & 1-\frac{2}{\pi} G \frac{\mu^{2}}{e}\left[\frac{7}{40}+\frac{1}{20} N_{g b}+\frac{1}{40} N_{d f}+\frac{1}{240} N_{m s}+\frac{1}{240} N_{c s}\right]+ \\
& \frac{1}{\pi} G \frac{e}{\mu^{2}}\left[\frac{7}{40}+\frac{1}{20} N_{g b}+\frac{1}{40} N_{d f}+\frac{1}{240} N_{m s}+\frac{1}{240} N_{c s}\right]\left(Q^{2}-\frac{\mu^{2}}{e}\right)^{2}+\ldots
\end{aligned}
$$

which under the narrow width approximation becomes

$$
\begin{aligned}
& 1-\frac{2}{\pi} G \frac{\mu^{2}}{e}\left[\frac{7}{40}+\frac{1}{20} N_{g b}+\frac{1}{40} N_{d f}+\frac{1}{240} N_{m s}+\frac{1}{240} N_{c s}\right]= \\
& \frac{1}{\pi} G \frac{e}{\mu^{2}}\left[\frac{7}{40}+\frac{1}{20} N_{g b}+\frac{1}{40} N_{d f}+\frac{1}{240} N_{m s}+\frac{1}{240} N_{c s}\right] \Delta^{2} \ll 1
\end{aligned}
$$

so that

$$
L_{r s}(p, k)=\tau_{\mu \nu} D^{\mu \nu \alpha \beta} \tau_{\alpha \beta} \longrightarrow \frac{20 \pi}{3} G \frac{\mu^{2}}{e} \frac{2 p^{2}+2 k^{2}-\mu^{2} / e-\left(p^{2}-k^{2}\right)^{2} e / \mu^{2}}{\Delta^{2}+\left(Q^{2}-\mu^{2} / e\right)^{2}}
$$

thus

$$
\beta\left(p^{2}\right)=\left.\int \frac{d^{4} k}{(2 \pi)^{4}} \frac{\beta\left(k^{2}\right)}{\alpha\left(k^{2}\right) k^{2}-\beta^{2} k^{2}} \tau_{\mu \nu} D^{\mu \nu \alpha \beta} \tau_{\alpha \beta}\right|_{(p-k)^{2}=Q^{2}}
$$




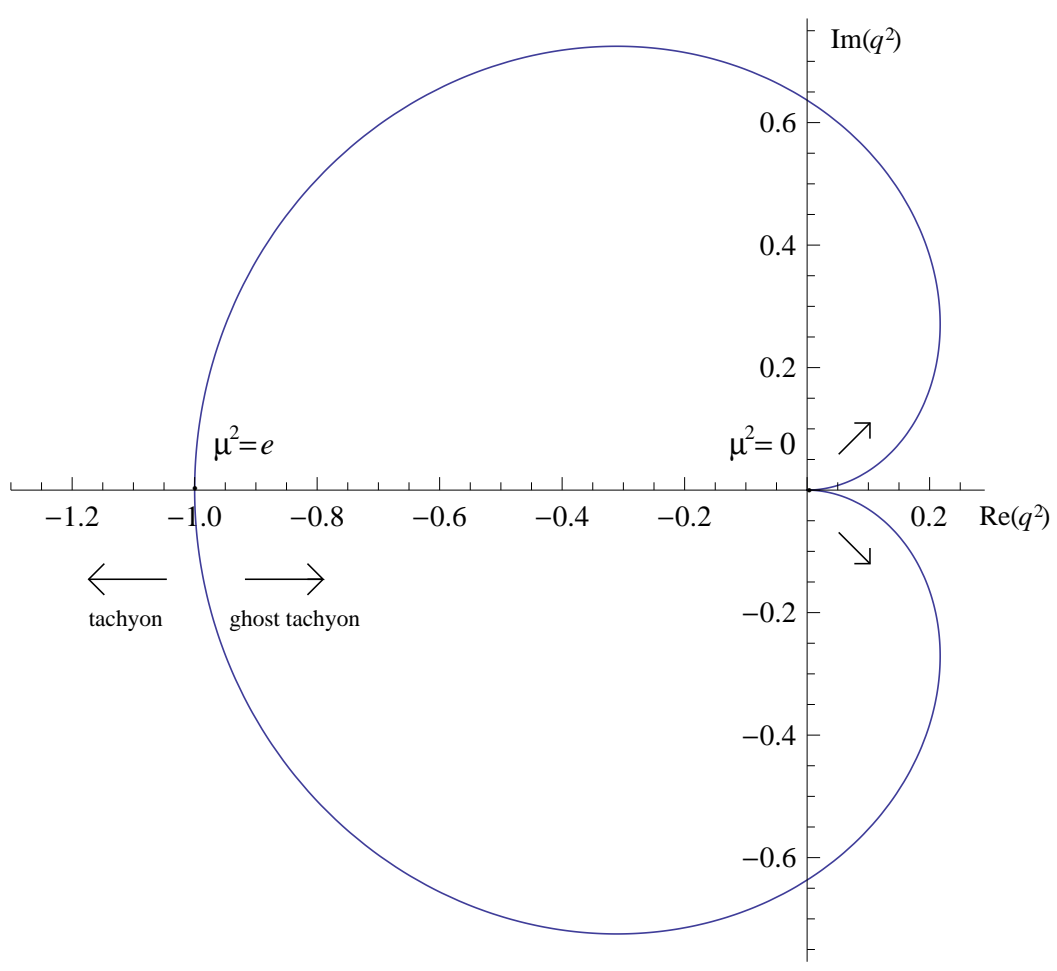

FIG. 8: The trajectory of the poles in the complex plane is shown as a function of the scale $\mu$ in units of the reduced Planck mass.

One can then perform the angular integration after the Wick rotation to find

$$
\begin{gathered}
\beta(x)=\frac{G^{2} \Lambda^{2} \mu^{2}}{16 \pi e} \int_{0}^{1} d y L_{r s}(x, y) \frac{y \beta(y)}{y \alpha^{2}(y)+\beta^{2}(y)} \\
L_{r s}(x, y)=\frac{5}{12}\left[\Delta-\sqrt[4]{\left(\Delta^{2}+\left(-R^{2}-2 \sqrt{x y}+x+y\right)^{2}\right)\left(\Delta^{2}+\left(-R^{2}+2 \sqrt{x y}+x+y\right)^{2}\right)}\right] \\
\left(\frac{2 x+2 y-R^{2}-(x-y)^{2} / R^{2}}{4 x y \Delta}\right) \cos \left[\frac{\operatorname{Arg}\left(2 x\left(-i \Delta+R^{2}+y\right)+\left(\Delta+i\left(R^{2}-y\right)^{2}\right)^{2}-x^{2}\right)}{2}\right]
\end{gathered}
$$

where $R^{2}=\mu^{2} / e$. Now, for a much smaller $N$, we can achieve symmetry breaking. Specifically, symmetry breaking is accomplished for

$$
\frac{7}{40}+\frac{1}{20} N_{g b}+\frac{1}{40} N_{d f}+\frac{1}{240} N_{m s}+\frac{1}{240} N_{c s}>3
$$

In Fig. 9 the solutions of the Schwinger-Dyson equation are shown for the resummed propagator under the narrow width approximation using an $N$ consistent with the matter content of supersymmetry (solid line). Despite being useful, as it allows to perform analitically the angular integration, the narrow width approximation does not capture the low momentum behaviour of the propagator and therefore offers a conservative estimate of the minimum matter content necessary to achieve dynamical symmetry breaking. A more accurate result can be obtained by numerically integrating the exact expression. The result is depicted in Fig. 9 in dashed line. In this case, slightly smaller matter contents also admit dynamical symmetry breaking.

\section{PURE LARGE $N$}

As we have seen the Schwinger-Dyson equations, which contain the full dynamical information of the quantum field gauge theory can serve as an adequate and effective tool for a non-perturbative approach to gravity. However, they 


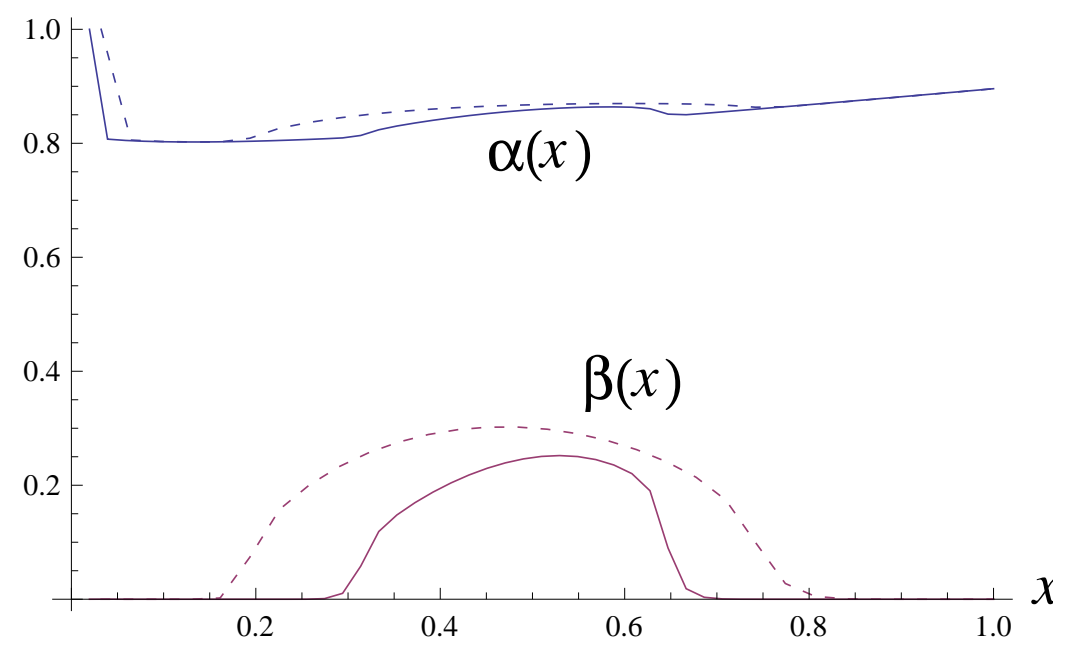

FIG. 9: The symmetry breaking solutions of the Schwinger-Dyson equation, $\alpha$ and $\beta$ after resummation as a function of $x=$ $p^{2} / \Lambda^{2}$ for a matter content consistent with supersymmetry using the narrow width approximation (solid line) and numerically integrating the exact expression (dashed line)

consist on an infinite chain of strongly coupled highly nonlinear integral equations, and some truncation scheme is needed in order to make these equations tractable for extracting physical information from them. At leading order, it is natural to assume that approximating the full vertices by their free perturbative (point-like) counterparts (and in addition replacing the full graviton propagator by its free perturbative expression) in the corresponding kernels of the above mentioned integral equations is consistent. Nonetheless it is known that such an approximation does not preserve explicit gravitational gauge covariance (general coordinate invariance).

Beyond the leading order, the matter contributions are separately gauge invariant at one loop and at large $N$. This is not clear for the gravitational loop contributions. There is a similar problem in QCD where the gluon loop contributions are partly canceled by vertex corrections. In this case it seems possible to take into account selfinteracting gluon modes (the non-Abelian character of QCD) at the level of the full gluon propagator only (the so called improved ladder approximation [13]), hence vertices remain intact, i.e. bare ones.

We will not follow this avenue here, instead we would like to consider the pure large $N$ regime, i.e. to consider in both the one loop calculation and the resummation only those contributions that exhibit the large $N$ enhancement and that are gauge invariant by themselves. Therefore we will eliminate the graviton loop contribution to the graviton self energy and assume that vertex or other contributions to the Schwinger-Dyson equations cancel the 't Hoof-Veltman contribution.

As we have seen, neither in the one loop nor in the resummed case, the graviton loop share to the kernel of the $\beta$ function is particularly important, therefore we do not expect our results regarding symmetry breaking to change in a significant way. However, the graviton loop was the key (together with the minimal scalar loop) to generate a conformal breaking term. Such a term controls the existence (and the magnitude) of the dynamically generated infrared mass. Then question is then, whether by suppressing the graviton loop, the infrared mass will disappear as well. The aswer to this question is no. The infrared mass could be generated by the known conformal anomalies of the matter fields - the trace anomalies. These would be suppressed by additional loop and coupling constant effects and will end up being roughly in the right ball-park to generate realistic right-handed neutrino masses.

We can now analyze the impact of the suppression of the graviton loop in the results obtained before. In the one loop calculation of the graviton propagator (in the large $N$ regime) we have seen that dynamical symmetry breaking is achieved for $A>8$. This remains being correct, except that (in the absence of minimal scalars) $A$ is now given by

$$
A=-\frac{12 N_{g b}+6 N_{d f}+N_{c s}}{288}
$$

and $C=0$. As a result, more matter fields are needed to compensate the missing contribution. Roughly 12 additional gauge bosons or 24 additional fermions.

In the resummed case, (in the absence of minimal scalars) the condition for symmetry breaking now reads

$$
\frac{1}{20} N_{g b}+\frac{1}{40} N_{d f}+\frac{1}{240} N_{c s}>3
$$


In this case just 7 additional fermions, or 4 additional gauge bosons will offset the graviton loop lost. In Fig. 10 the exact solutions of the Schwinger-Dyson equation are shown for the resummed propagator using an $N$ consistent with the matter content of supersymmetry in the pure large $N$ regime (solid line). For comparison, the solutions including the graviton loop contribution to the graviton self energy are also shown (dashed line).

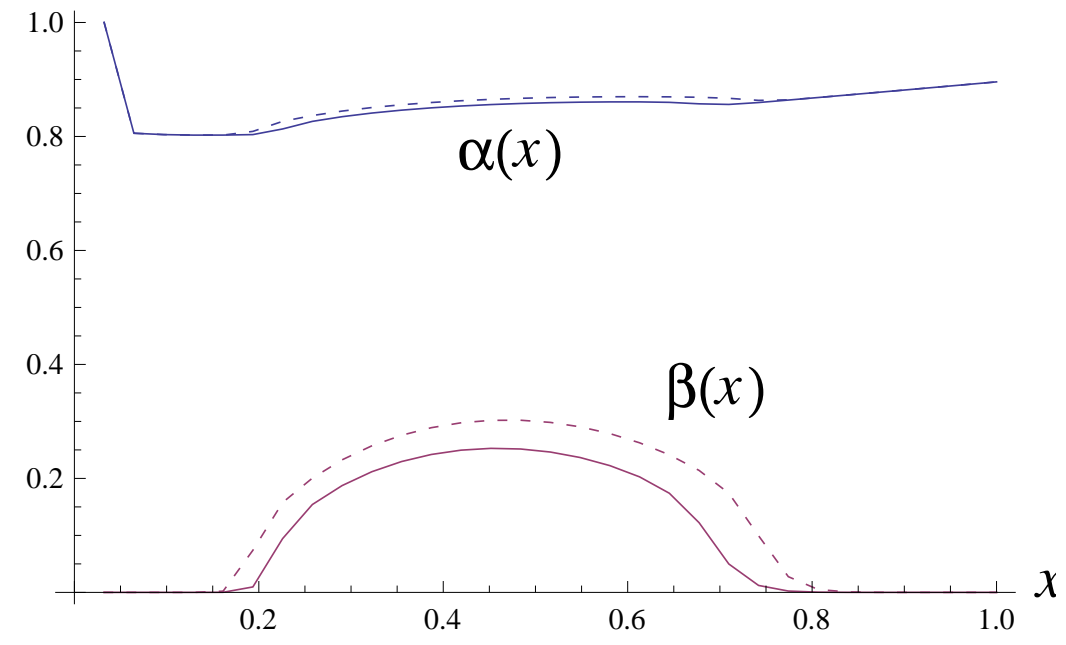

FIG. 10: The exact symmetry breaking solutions of the Schwinger-Dyson equation, $\alpha$ and $\beta$ after resummation as a function of $x=p^{2} / \Lambda^{2}$ for a matter content consistent with supersymmetry with (dashed line) and without (solid line)the graviton loop input

\section{DISCUSSION AND CONCLUSIONS}

In this work we studied the possibility that an enhanced gravitational attraction can spark the formation of a right handed neutrino condensate, inducing dynamical symmetry breaking and generating a Majorana mass for the right handed neutrino at a scale appropriate for the see-saw mechanism. The composite field formed by the condensate phase could drive an early epoch of inflation leaving an imprint tightly constrained, that can be experimentally probed in the near future.

The major task in studying chiral symmetry breaking is to establish the Schwinger-Dyson equation for the fermion self-energy, which takes into account the non-perturbative features of the theory and then, to investigate whether this equation admits a non-zero fermion mass as a solution.

We have found that to the lowest order, the theory does not allow dynamical symmetry breaking. Nevertheless, thanks to the large number of matter fields in the standard model (and the ever larger one in extended models) the suppression by an additional power in $G$ in the next leading term can be compensated, enhancing these higher order terms up to their lowest order counterparts. We have seen then, that for a vast number of matter fields chiral symmetry can be broken dynamically and the infrared mass generated this way turns out to be in the expected range for a successful see-saw scenario.

To further exploit the large $N$ potential, we considered a $1 / N$ expansion of the graviton propagator, keeping $G N$ fixed. The resulting lowest order graviton propagator has $1 /\left(q^{4} \log \left(q^{2}\right)\right)$ asymptotic behaviour and no tachyon or unphysical real bound state poles. There are however, complex conjugate poles on the physical sheet that when close to the real axis, significatively enhance the spacelike propagator. This enhancement allows a dynamical symmetry breaking solution for a matter content consistent with the standard model.

A distinguishing feature of the model is the presence of non-local effects associated with the complex poles. Such effects become appreciable only at ultra high energy scales. It will be quite interesting to investigate the effects of these nonlocalities in the very early universe where they become relevant, in particular with respect to the horizon and homogeneity problems.

Probably the least attractive feature of our model is the fact that we are working at energies close to Planck scale, where our physics knowledge is rather poor. It is widely believed that field theory breaks down at Planck scale because gravity becomes important and a full theory of quantum gravity is called for. If the gauge force strength is characterized by the dimensionless gravitational coupling $\alpha_{G}=G^{2} \Lambda^{2}=\Lambda^{2} / M_{P l}^{2}$ at an energy scale $\Lambda$, then yet 
unknown quantum gravity effects become dominant at energy scales close to $M_{P l}$ where $\alpha_{G}$ approaches 1 , the regime needed for dynamical symmetry breaking. However, since gravity (and thus the graviton) couples to all fields, matter loop corrections can enhance the effective gravitational coupling to the needed range, before full quantum gravity completely blurs the picture.

In our case, it is crucial to notice that there are (at least) three relevant scales, one fundamental scale, the original Planck scale, and two dynamically induced scales, the reduced Planck scale, $M_{P l \text { reduced }}^{2}=M_{P l}^{2} / N$, that governs size of the net matter loop corrections and the scale where the dominant chiral symmetry breaking occurs for the condensate. Of course, there is also the scale of the infrared mass which is suppressed relative to the others scales and is relevant for the phenomenology of the right-handed neutrinos. The reason for this suppression is that the symmetry breaking is largely due to the $A$ term, which is the dominant contribution to the kernel for the $\beta$ function, while the infrared mass is generated by the conformal breaking $C$ term, which does not enjoy the large $N$ enhancement.

As we have seen the dynamical scales are suppressed relative the the original Planck scale courtesy of the large matter content of the model, which implies an inevitable hierarchy between the energy scale of the field theory and the Planck scale. This effectively amounts to an enhancemnet of the dimensionless gravitational coupling, $\alpha_{G \text { large } N}=N \alpha_{G}$ at momenta that are below the normal Planck scale opening the door to dynamical symmetry breaking at those scales. We assume that as the energies involved are sufficiently below $M_{P l}$, we are not sensitive to black hole formation and other nonlinear effects which could dominate Planck scale physics.

Before closing, we would like to mention that there is a net gravitational anomaly for the singlet chiral current associated with the right-handed neutrino phases (gravitational anomalies cancel for the Standard Model gauge currents as $\operatorname{Tr}(Y)=0$ ). If the condensate is the only breaking of these phases then there should be massless NambuGoldstone Bosons (NGBs), or massive pseudoNGBs if there is some other explicit breaking. These NGBs or pseudo NGBs are our would-be inflatons. Despite being a kind of explicit breaking, the gravitational anomaly does not necessarily generate a mass. Instantons make the $\eta^{\prime}$ heavy in QCD but the electroweak anomalies make little or no contribution to masses. Even if the anomaly does not generate a mass for the PNGB, it may still have an important role in our model model as it could play a role ending the inflationary phase.

\section{ACKNOWLEDGMENTS}

I am deeply grateful and pleased to acknowledge Bill Bardeen, with whom I am specially indebted, for his support, encouragement, and guidance during this work (and for his invaluable patience with me and my manuscripts). Financial support from Spanish MEC and FEDER (EC) under grant FPA2008-02878, and Generalitat Valenciana under the grant PROMETEO/2008/004 is also acknowledged. Feynman diagrams were made using Jaxodraw [14]

\section{Appendix A: Feynman rules}

We list here the Feyman rules which are employed in our calculations. For derivations of these forms, see [15] The propagator for a massless fermion field is given by

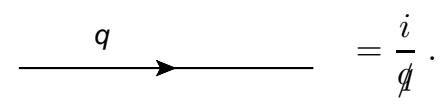

In harmonic (Feynman) gauge the graviton propagator has been discussed in [6, 16].

$$
\text { 几ح } \sim \alpha \beta=\frac{i \mathcal{P}^{\mu \nu \alpha \beta}}{q^{2}}
$$

where the polarization sum is given by

$$
\mathcal{P}^{\mu \nu \alpha \beta}=\frac{1}{2}\left(\eta^{\mu \alpha} \eta^{\nu \beta}+\eta^{\mu \beta} \eta^{\nu \alpha}-\eta^{\mu \nu} \eta^{\alpha \beta}\right)
$$

The 1-graviton-2-(massless)fermion vertex is given by

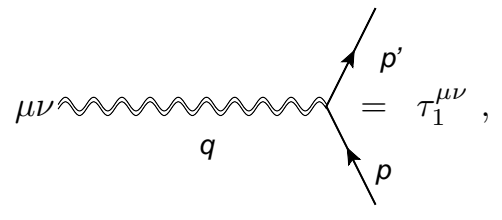


with

$$
\tau_{1}^{\mu \nu}=\frac{i \kappa}{2}\left[\left(p^{\prime}+p\right)_{\mu} \gamma_{\nu}+\left(p^{\prime}+p\right)_{\nu} \gamma_{\mu}-\eta_{\mu \nu}\left(\not p^{\prime}+\not p\right)\right]
$$

The 1-graviton-2-scalar vertex reads

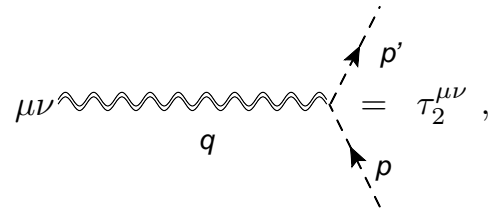

with

$$
\tau_{2}^{\mu \nu}=\frac{-i \kappa}{2}\left\{p^{\mu} p^{\prime \nu}+p^{\nu} p^{\prime \mu}-\eta^{\mu \nu}\left(p \cdot p^{\prime}\right)\right\}
$$

The 1-graviton-2-photon vertex is given by

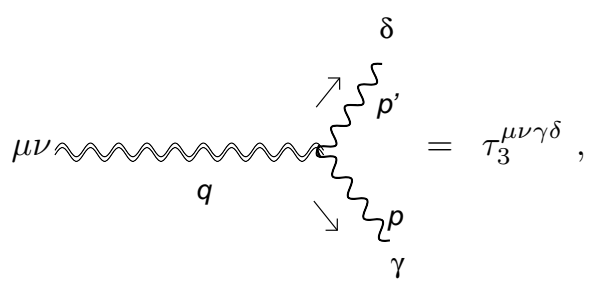

with

$$
\begin{aligned}
\tau_{3}^{\mu \nu \gamma \delta} & =i \kappa\left\{\mathcal{P}^{\mu \nu \gamma \delta}\left(p \cdot p^{\prime}\right)+\frac{1}{2}\left[\eta^{\mu \nu} p^{\delta} p^{\prime \gamma}+\eta^{\gamma \delta}\left(p^{\mu} p^{\prime \nu}+p^{\nu} p^{\prime \mu}\right)-\left(\eta^{\mu \delta} p^{\prime \gamma} p^{\nu}+\eta^{\nu \delta} p^{\prime \gamma} p^{\mu}\right.\right.\right. \\
& \left.\left.\left.+\eta^{\nu \gamma} p^{\prime \mu} p^{\delta}+\eta^{\mu \gamma} p^{\prime \nu} p^{\delta}\right)\right]\right\} .
\end{aligned}
$$

Using the background field method the 3-graviton vertex is found as [16]

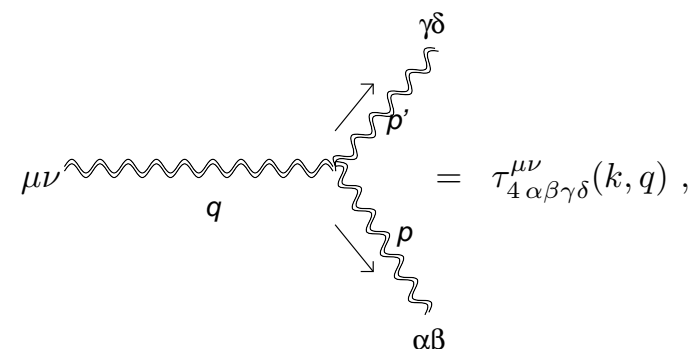

with

$$
\begin{aligned}
\tau_{4 \alpha \beta \gamma \delta}^{\mu \nu}=-\frac{i \kappa}{2} & \cdot\left\{\mathcal{P}_{\alpha \beta \gamma \delta}\left[k^{\mu} k^{\nu}+(k-q)^{\mu}(k-q)^{\nu}+q^{\mu} q^{\nu}-\frac{3}{2} \eta^{\mu \nu} q^{2}\right]+2 q_{\lambda} q_{\sigma}\left[\mathbb{1}_{\alpha \beta}{ }^{\sigma \lambda} \mathbb{1}_{\gamma \delta}{ }^{\mu \nu}+\mathbb{1}_{\gamma \delta}{ }^{\sigma \lambda} \mathbb{1}_{\alpha \beta}{ }^{\mu \nu}-\mathbb{1}_{\alpha \beta}{ }^{\mu \sigma} \mathbb{1}_{\gamma \delta}{ }^{\nu \lambda}\right.\right. \\
& \left.-\mathbb{1}_{\gamma \delta}{ }^{\mu \sigma} \mathbb{1}_{\alpha \beta}{ }^{\nu \lambda}\right]+\left[q_{\lambda} q^{\mu}\left(\eta_{\alpha \beta} \mathbb{1}_{\gamma \delta}{ }^{\nu \lambda}+\eta_{\gamma \delta} \mathbb{1}_{\alpha \beta}{ }^{\nu \lambda}\right)+q_{\lambda} q^{\nu}\left(\eta_{\alpha \beta} \mathbb{1}_{\gamma \delta}{ }^{\mu \lambda}+\eta_{\gamma \delta} \mathbb{1}_{\alpha \beta}{ }^{\mu \lambda}\right)\right. \\
& \left.-q^{2}\left(\eta_{\alpha \beta} \mathbb{1}_{\gamma \delta}{ }^{\mu \nu}+\eta_{\gamma \delta} \mathbb{1}_{\alpha \beta}{ }^{\mu \nu}\right)-\eta^{\mu \nu} q_{\lambda} q_{\sigma}\left(\eta_{\alpha \beta} \mathbb{1}_{\gamma \delta}{ }^{\sigma \lambda}+\eta_{\gamma \delta} \mathbb{1}_{\alpha \beta}{ }^{\sigma \lambda}\right)\right] \\
& +\left[2 q_{\lambda}\left\{\mathbb{1}_{\alpha \beta}{ }^{\lambda \sigma} \mathbb{1}_{\gamma \delta \sigma}{ }^{\nu}(k-q)^{\mu}+\mathbb{1}_{\alpha \beta}{ }^{\lambda \sigma} \mathbb{1}_{\gamma \delta \sigma}{ }^{\mu}(k-q)^{\nu}-\mathbb{1}_{\gamma \delta}{ }^{\lambda \sigma} \mathbb{1}_{\alpha \beta \sigma}{ }^{\nu} k^{\mu}-\mathbb{1}_{\gamma \delta}{ }^{\lambda \sigma} \mathbb{1}_{\alpha \beta \sigma}{ }^{\mu} k^{\nu}\right\}\right. \\
& \left.+q^{2}\left(\mathbb{1}_{\alpha \beta \sigma}{ }^{\mu} \mathbb{1}_{\gamma \delta}{ }^{\nu \sigma}+\mathbb{1}_{\alpha \beta}{ }^{\nu \sigma} \mathbb{1}_{\gamma \delta \sigma}{ }^{\mu}\right)+\eta^{\mu \nu} q_{\sigma} q_{\lambda}\left(\mathbb{1}_{\alpha \beta}{ }^{\lambda \rho} \mathbb{1}_{\gamma \delta \rho}{ }^{\sigma}+\mathbb{1}_{\gamma \delta}{ }^{\lambda \rho} \mathbb{1}_{\alpha \beta \rho}{ }^{\sigma}\right)\right]+\left\{\left(k^{2}+(k-q)^{2}\right)\right. \\
& \left.\left.\cdot\left[\mathbb{1}_{\alpha \beta}{ }^{\mu \sigma} \mathbb{1}_{\gamma \delta \sigma}{ }^{\nu}+\mathbb{1}_{\gamma \delta}{ }^{\mu \sigma}{ } \mathbb{1}_{\alpha \beta \sigma}{ }^{\nu}-\frac{1}{2} \eta^{\mu \nu} \mathcal{P}_{\alpha \beta \gamma \delta}\right]-\left(\mathbb{1}_{\gamma \delta}{ }^{\mu \nu} \eta_{\alpha \beta} k^{2}-\mathbb{1}_{\alpha \beta}{ }^{\mu \nu} \eta_{\gamma \delta}(k-q)^{2}\right)\right\}\right\}
\end{aligned}
$$

and

$$
\mathbb{1}_{\alpha \beta \gamma \delta}=\frac{1}{2}\left(\eta_{\alpha \gamma} \eta_{\beta \delta}+\eta_{\alpha \delta} \eta_{\beta \gamma}\right)
$$


a complete set of Feynman rules can be found in [17, 18].

[1] E. W. Kolb and M. S. Turner, "The Early universe," Front. Phys. 69 (1990) 1.

S. Dodelson, "Modern Cosmology," Amsterdam, Netherlands: Academic Pr. (2003) $440 p$

[2] A. H. Guth, Phys. Rev. D 23 (1981) 347.

A. D. Linde, Phys. Lett. B 108 (1982) 389.

A. Albrecht and P. J. Steinhardt, Phys. Rev. Lett. 48 (1982) 1220.

[3] P. Minkowiski, Phys. Lett. B 67, 421 (1977).

M. Gell-Mann, P. Ramond and R. Slansky in Supergravity, eds.D. Freedman and P. Van Niuenhuizen (North Holland, Amsterdam, 1979),p. 315.

T. Yanagida in Proceedings of the Workshop on Unified Theory and Baryon Number in the Universe, eds. O. Sawada and A. Sugamoto (KEK, Tsukuba, Japan, 1979).

[4] G. Barenboim, JHEP 0903 (2009) 102 arXiv:0811.2998 [hep-ph]].

[5] K. Freese, J. A. Frieman and A. V. Olinto, Phys. Rev. Lett. 65 (1990) 3233.

F. C. Adams, J. R. Bond, K. Freese, J. A. Frieman and A. V. Olinto, Phys. Rev. D 47 (1993) 426 arXiv:hep-ph/9207245.

[6] G. 't Hooft and M. Veltman. One-loop diverencies in the theory of gravitation. Ann. Inst. Henri Poincaré, Sec. A, 20(1):69-94, 1974.

[7] O. Abe and Y. Hoshino, Stability Of Fermion And Momentum Cutoff In Quantum Gravity In Flat Background Space-Time. Phys. Lett. B 139 (1984) 167.

[8] E. Tomboulis, Phys. Lett. B 70 (1977) 361

[9] E. Tomboulis, Phys. Lett. B 97 (1980) 77.

[10] T. D. Lee and G. C. Wick, Nucl. Phys. B 9 (1969) 209. Phys. Rev. D 3 (1971) 1046. Phys. Rev. D 2 (1970) 1033.

[11] J. M. Cornwall, R. Jackiw and E. Tomboulis, Phys. Rev. D 10 (1974) 2428.

[12] J. D. Lykken, arXiv:hep-th/9612114

[13] V.A.Miransky, "Dynamical Symmetry Breaking in Quantum Field Teories", Word Scientific, Singapore, 1993; K.Higashijima, Prog. Theor. Phys. Suppl., 104 (1991) 1-69.

[14] D. Binosi and L. Theussl, Comput. Phys. Commun. 161 (2004) 76 arXiv:hep-ph/0309015.

[15] N. E. J. Bjerrum-Bohr. Cand. Scient. thesis. University of Copenhagen, 2001.

[16] J. F. Donoghue. General relativity as an effective field theory: The leading quantum corrections. Phys. Rev. D, 50(6):38743888, 1994. [arXiv:gr-qc/9305057v1].

[17] N. E. J. Bjerrum-Bohr et al. Quantum gravitational corrections to the nonrelativistic scattering potential of two masses. Phys. Rev. D, 67(084033):1-12, 2003. [arXiv:hep-th/0211072], Erratum-ibid. D71, 069903 (2005).

[18] S. Faller, Phys. Rev. D 77 (2008) 124039 arXiv:0708.1701 [hep-th]]. 